\title{
COUNTABLE BOX PRODUCTS OF ORDINALS
}

\author{
BY \\ MARY ELLEN RUDIN
}

\begin{abstract}
The countable box product of ordinals is examined in the paper for normality and paracompactness. The continuum hypothesis is used to prove that the box product of countably many o-compact ordinals is paracompact and that the box product of another class of ordinals is normal. A third class trivially has a nonnormal product.
\end{abstract}

Because I have found a countable box product of ordinals useful in the past [1], this class of spaces particularly interests me. The purpose of this paper is to tell what I know about which of these spaces is paracompact or normal.

In [2] I prove that the continuum hypothesis implies the box product of countably many $\sigma$-compact, locally compact, metric spaces is paracompact. I prove here that the continuum hypothesis implies the box product of countably many $\sigma$-compact ordinals is paracompact (Theorem 1) and the box product of another class of ordinals is normal (Theorem 2). The proof of Theorems 1 and 2 is a quite messy join of the techniques of [1] and [2] which raises some doubt in my mind as to whether these theorems are worth proving. Because I care, because I think these spaces are set theoretically interesting and topologically useful, because I think these theorems are best possible, the theorems are worth the mess to me.

A. If $\left\{X_{\lambda}\right\}_{\lambda \in \Lambda}$ is a family of topological spaces, a box in $\Pi_{\lambda \in \Lambda} X_{\lambda}$ is a set $\Pi_{\lambda \in \Lambda} U_{\lambda}$ where each $U_{\lambda}$ is open in $X_{\lambda}$. The box product of $\left\{X_{\lambda}\right\}_{\lambda \in \Lambda}$ is $\prod_{\lambda \in \Lambda} X_{\lambda}$ topologized by using the set of all boxes in it as a basis.

Throughout the paper the following notation is used.

An ordinal $\alpha$ is the set of all ordinals less than $\alpha$ and $\alpha$ is topologized by the interval topology. The statement that $\alpha$ is a cardinal means that $\alpha$ is an ordinal and no smaller ordinal has the same cardinality as $\alpha$.

The notation $\prod_{\lambda \in \Lambda} \beta_{\lambda}$ is used to mean the ordinary Cartesian product of the $\beta_{\lambda}$ 's and never the cardinal or ordinal arithmetic product. Similarly $\alpha^{\beta}$ means the set of all functions from $\beta$ into $\alpha$ rather than an arithmetic operation.

If $\alpha$ is an ordinal, let $\operatorname{cf}(\alpha)$ denote the cofinality of $\alpha$; that is $\operatorname{cf}(\alpha)$ is the smallest ordinal $\delta$ such that there is a subset $\Delta$ of $\alpha$, order isomorphic with $\delta$, such that $\beta<\alpha$ implies there is a $\gamma \in \Delta$ with $\beta \leq \gamma$. Observe that $\alpha$ is a $\sigma$-compact ordinal if and only if $\alpha$ is compact or $\operatorname{cf}(\alpha)=\omega_{0}$.

Received by the editors December 10, 1971 and, in revised form, October 1, 1972.

AMS (MOS) subject classifications (1970). Primary 54B10, 54A25, 54D15, 54D20, 54D30, 02K25.

Key words and phrases. Product, box product, continuum hypothesis, paracompact, normal, $\theta$ compact.

Copyright $\odot$ 1974, American Mathematical Society 
Let $[\mathrm{CH}]$ and $[\mathrm{GCH}]$ denote the continuum hypothesis is true and the generalized continuum hypothesis is true, respectively.

To avoid repetition assume that for each $n \in \omega_{0}, \alpha_{n}$ is a positive ordinal, and let $X$ be the box product of $\left\{\alpha_{n}\right\}_{n \in \omega_{0}}$. If $x \in X$ or $U \subset X$, let $x(n)$ and $U(n)$ denote the projection of $x$ and $U$, respectively, on $\alpha_{n}$.

B. Let $S=\left\{n \in \omega_{0} \mid \alpha_{n}\right.$ is not $\sigma$-compact $\}$.

Case 0 . The trivial case. There are $n \in S$ and $m \in \omega_{0}-\{n\}$ with $\alpha_{m}>\operatorname{cf}\left(\alpha_{n}\right)$. Theorem 0 yields $X$ is not normal.

Case 1. The basic case. $S=\varnothing$. Theorem 1 yields $[\mathrm{CH}] X$ is paracompact.

Case 2. The other case. Not Cases 0 or 1.

Since not Case $1, S \neq \varnothing$. And $s \in S$ imply $\alpha_{s}$ is not paracompact; so $X$ is not paracompact. Define $\kappa(X)=\sup \left\{\right.$ cardinality of $\prod_{n \in \omega_{0}-\{s\}} \beta_{n} \mid s \in S, \beta_{n} \leq \alpha_{n}$, and $\beta_{n}$ is compact $\}$. Since $S \neq \varnothing$ and not Case 0 , there is a unique uncountable $\delta$ such that $\operatorname{cf}\left(\alpha_{s}\right)=\delta$ for all $s \in S$. Since not Case 0 and $\delta$ is uncountable, $\kappa(X) \leq \sup \left\{\right.$ cardinality of $\beta^{\omega_{0}} \mid \beta$ is a cardinal less than $\delta$ \}. Thus [GCH] implies $\kappa(X)<\delta$ unless $\delta$ is the cardinal successor of an infinite $\sigma$-compact cardinal. Theorem 2 proves $\kappa(X)<\delta$ and $[\mathrm{CH}]$ implies $X$ is normal.

Thus in Case 2, if $\delta$ is not the cardinal successor of an infinite $\sigma$-compact cardinal, then $[\mathrm{GCH}] X$ is normal but not paracompact.

I give some examples to show the flavor of the results.

(1) $\alpha_{0}=\omega_{2}$ and $\alpha_{n}=\omega_{0}+1$ for $n>0$; [CH]X is normal; Case 2.

(2) $\alpha_{n}=\omega_{k}$ for a finite $k>1$ and all $n ;[\mathrm{CH}] X$ is normal; Case 2.

(3) $\alpha_{n}=\omega_{\omega_{0}+2}$ for $n$ even and $\alpha_{n}=\omega_{0}+1$ for $n$ odd; [GCH] $X$ is normal; Case 2.

(4) $\alpha_{n}=\omega_{n}$ for all $n ; X$ is not normal; Case 0.

(5) $\alpha_{n}=\omega_{\omega_{0}}$ for all $n ;[\mathrm{CH}] X$ is paracompact; Case 1 .

(6) $\alpha_{n}=\omega_{0}+1$ for all $n ;[\mathrm{CH}] X$ is paracompact; Case 1 .

But I conjecture there is a model of set theory in which $X$ is not normal.

(7) $\alpha_{0}=\omega_{1}$ and $\alpha_{n}=\omega_{0}+1$ for all $n>0$; Case 2 but none of the present results apply. I conjecture $X$ is not normal in a model of set theory including [CH]. $I$ also conjecture $X$ is normal in a different model of set theory.

C. Let $T=\left\{t \in \omega_{0} \mid \alpha_{t}\right.$ is $\sigma$-compact but not compact $\}$.

Lemma 0. Without loss of generality, $T=\varnothing$.

Proof. If $t \in T$, choose nonlimit ordinals $\alpha_{0 t}<\alpha_{1 t}<\cdots$ having $\alpha_{t}$ as a limit. Define $\mathbb{Q}=\left\{\prod_{n \in \omega_{0}} A_{n} \mid A_{n}=\alpha_{n}\right.$ if $n \in \omega_{0}-T$, and either $A_{n}=\alpha_{0 n}$ or $A_{n}$ $=\alpha_{i n}-\alpha_{(i-1) n}$ for some $i>0$ if $\left.n \in T\right\}$. Clearly $\mathbb{Q}$ is a collection of disjoint open sets covering $X$; thus $X$ is paracompact (normal) if and only if all members of $\mathbb{Q}$ are paracompact (normal). But $\prod_{n \in \omega_{0}} A_{n} \in \mathbb{Q}$ implies $A_{n}$ is either isomorphic to a compact ordinal or is a non- $\sigma$-compact ordinal. Hence we may assume $T=\varnothing$. 
D.

Theorem 0. Suppose $\gamma$ and $\beta$ are ordinals; $\gamma$ is not $\sigma$-compact, and $\beta>\operatorname{cf}(\gamma)$. Then $\gamma \times \beta$ is not normal.

Proof. Let $\delta=\operatorname{cf}(\gamma)$. Since $\delta \times(\delta+1)=D$ is homeomorphic to a closed subset of $\gamma \times \beta$, it suffices to show that $D$ is not normal. For $\alpha<\delta$, let $h_{\alpha}=(\alpha, \delta)$ and $k_{\alpha}=(\alpha, \alpha)$. Then $H=\left\{h_{\alpha} \mid \alpha<\delta\right\}$ and $K=\left\{k_{\alpha} \mid \alpha<\delta\right\}$ are disjoint closed subsets of $D$. Suppose $U$ and $V$ are disjoint sets open in $D$ and $U \supset H$ and $V \supset K$. Let $\Lambda=\{\lambda<\delta \mid \lambda$ is a limit ordinal $\}$. Since $k_{\lambda} \in V$ for each $\lambda \in \Lambda$, there is a $\beta_{\lambda}<\lambda$ with $\left\{(\eta, \lambda) \mid \beta_{\lambda} \leq \eta \leq \lambda\right\} \subset V$. Since $\operatorname{cf}(\delta)=\delta$, there is a $\beta<\delta$ such that $\rho<\delta$ implies $\left\{\lambda \in \Lambda \mid \beta_{\lambda}=\beta\right.$ and $\left.\lambda>\rho\right\} \neq \varnothing$. Since $h_{\beta} \in U$, there is a $\rho<\delta$ such that $\{(\beta, \lambda) \mid \rho \leq \lambda \leq \delta\} \subset U$. Choose $\lambda \in \Lambda$ such that $\beta_{\lambda}=\beta$ and $\lambda \geq \rho$. Then $(\beta, \lambda) \in U \cap V$. Thus $X$ is not normal.

Conjecture based on the proof of Theorem 0 . Let $\alpha_{0}=\omega_{1}$ and $\alpha_{n}=\omega_{0}+1$ for $n>0$. For $\alpha<\omega_{1}$ define $h_{\alpha} \in X$ by $h_{\alpha}(0)=\alpha$ and $h_{\alpha}(n)=\omega_{0}$ for $n>0$. For $\alpha<\omega_{1}$ choose $k_{\alpha} \in X$ so $k_{\alpha}(0)=\alpha$ and, for $n>0$, choose $k_{\alpha}(n)<\omega_{0}$ in such a way that $\beta<\alpha$ implies there is an $m \in \omega_{0}$ so $n>m$ gives $k_{\alpha}(n)>k_{\beta}(n)$. Then $H=\left\{h_{\alpha} \mid \alpha<\omega_{1}\right\}$ and $K=\left\{k_{\alpha} \mid \alpha<\omega_{1}\right\}$ are again closed and disjoint subsets of $X$. I conjecture that in some model of set theory including $[\mathrm{CH}], K$ can be chosen in such a way that $H$ and $K$ cannot be separated. However, in a model with no scale of cardinality $\aleph_{1}$ (that is, for any $K$ there is an $x \in \omega_{0} \times \omega_{0} \times \cdots$ such that for all $\alpha$ there is an $m$ for which $n>m$ implies $\left.x(n)>k_{\alpha}(n)\right)$ I feel $X$ must be normal. $[\mathrm{CH}]$ implies there is a scale of cardinality $x_{1}$.

E. The theorems are proved in this section and we need more notation. Assume $\alpha_{n}$ is compact for all $n$.

Let $\mathcal{L}=\left\{\prod_{n \in \omega_{0}} J_{n} \mid J_{n}\right.$ is a closed subinterval of $\left.\alpha_{n}\right\}$; we allow $J_{n}=\varnothing$. See that $X \in \mathcal{L}$ and the intersection of the members of any subset of $\mathcal{L}$ is a member of $\mathcal{L}$. If $L \in \mathscr{L}$, define $\prod_{n \in \omega_{0}} \sup \{p(n) \mid p \in L\}$ to be the top of $L$; observe that $\varnothing \neq L \in \mathcal{L}$ implies the top of $L$ belongs to $L$.

Let $\mathscr{B}=\{U \in \mathcal{L} \mid U(n)$ is both open and closed for all $n\}$; $\mathscr{B}$ is a basis for the topology of $X$.

For $p \in X$ and $n \in \omega_{0}$, let $E^{n}(p)=\{x \in X \mid x(m)=p(m)$ for all $m \geq n\}$. Let $E(p)=\cup_{n \in \omega_{0}} E^{n}(p)$ and $\mathcal{E}=\left\{\cup_{p \in U} E(p) \mid U \in \mathscr{B}\right\}$. For $V \in \delta$ let $V^{*}$ denote a particular $U \in \mathscr{B}$ with $V=\cup_{p \in U} E(p)$. Observe that the relation $E$, where $p E q$ means $p(m)=q(m)$ for all but finitely many $m \in \omega_{0}$, partitions $X$ into equivalence classes and that $E(p)$ is the equivalence class to which $p$ belongs. Also $V \in \mathcal{E}$ implies

$$
V=\bigcup_{n \in \omega_{0}} \alpha_{0} \times \alpha_{1} \times \cdots \times \alpha_{n-1} \times V^{*}(n) \times V^{*}(n+1) \times \cdots .
$$

From this we see that $V \in \mathcal{E}$ implies $V$ is the union of disjoint members of $\mathscr{B}$ 
because $V=\cup_{n \in \omega_{0}}\left\{\prod_{j \in \omega_{0}} J_{j} \mid J_{j}(i)=V^{*}(i)\right.$ for $i \geq n$ and $J_{j}(i)$ is either $V^{*}(i)$ or a maximal subinterval of $\left.\alpha_{n}-V^{*}(i)\right\}$.

Let $\Omega$ be the set of all subsets $\mathscr{Q}$ of $\mathscr{B}$ covering $X$ such that $V \in \mathbb{Q}$ and $U \in \mathscr{B}$ and $V \supset U$ imply $U \in \mathbb{Q}$. For $\mathbb{Q} \in \Omega$ define $\mathcal{E}(\mathbb{Q})=\{V \in \mathcal{E} \mid V$ is the union of a set of disjoint members of $\mathbb{Q}$.

We now prove a sequence of lemmas.

Lemma 1. The intersection of the members of a countable subset of $\mathcal{E}$ is the union of a set of disjoint members of $\mathcal{E}$.

Proof. Assume $\left\{V_{n}\right\}_{n \in \omega_{0}} \subset \mathcal{E}$.

For $m$ and $n$ in $\omega_{0}$ let $g_{m n}=\left\{I \mid I\right.$ is either $V_{m}^{*}(n)$ or a maximal interval in $\left.\alpha_{n}-V_{m}^{*}(n)\right\}$. Observe that $g_{m n}$ partitions $\alpha_{n}$ into three or fewer disjoint open and closed subintervals. Define $g_{n}=\left\{\cap_{m \leq n} I_{m} \mid I_{m} \in g_{m n}\right\}$. Let $g=\left\{\prod_{n \in \omega_{0}} J_{n} \mid J_{n}\right.$ $\left.\in g_{n}\right\}$. Finally let $\mathscr{K}=\left\{\cup_{p \in J} E(p) \mid J \in \mathcal{G}\right\}$ and $\mathcal{K}=\left\{V \in \mathscr{K} \mid V \subset \bigcap_{n \in \omega_{0}} V_{n}\right\}$. Since the terms of $g_{n}$ are disjoint open and closed intervals of $\alpha_{n}$ whose union is $\alpha_{n}$, the terms of $\mathcal{K}$ are disjoint members of $\mathcal{E}$ whose union is $X$.

Suppose $x \in \bigcap_{n \in \omega_{0}} V_{n}$; choose $k_{0}<k_{1}<\cdots$ in $\omega_{0}$ so $x(n) \in V_{m}^{*}(n)$ for all $n \geq k_{m}$. Define $J_{n}$ by $x(n) \in J_{n} \in g_{n}$. Since $k_{m} \geq m$, clearly $J_{n} \subset V_{m}^{*}(n)$ for $n \geq k_{m}$. Let $J=\prod_{n \in \omega_{0}} J_{n}$ and $V=\cup_{p \in J} E(p)$. Then $x \in V \in \Re_{0}$ Asssume $y \in V$ and $m \in \omega_{0}$. There is a $k \in \omega_{0}$ so $y(n) \in J_{n}$ for all $n>k$; so $y(n)$ $\in V_{m}^{*}(n)$ for all $n>k_{m}+k$. Choose a point $p$ of $V_{m}^{*}$ such that $p(n)=y(n)$ for $n>k_{m}+k$; then $y \in E(p)$ so $y \in V_{m}$. Thus $V \in \mathcal{T}$. Hence $\mathcal{H}$ is a set of disjoint members of $\delta$ whose union is $\bigcap_{n \in \omega_{0}} V_{n}$.

Lemma 2. If $x \in X$ and $\mathbb{Q} \in \Omega$, then $x \in \cup \mathcal{E}(\mathbb{Q})$.

Proof. By induction we define for each $n \in \omega_{0}$ a finite subset $\mathbb{Q}_{n}$ of $\mathbb{Q}$ such that $V \in \mathbb{Q}_{n}$ implies $V \cap E^{n}(x) \neq \varnothing ; \mathbb{Q}_{n}$ is a cover of $E^{n}(x)$ by disjoint sets. Also there is an open-closed interval $I_{n}$ of $\alpha_{n}$ to which $x(n)$ belongs which is $V(n)$ for all $V \subset \mathbb{Q}_{n}$.

Choose $W \in \mathbb{Q}$ such that $x \in W$ and let $\mathbb{Q}_{0}=\{W\}$ and $I_{0}=W(0)$.

Assume $\mathbb{Q}_{n-1}$ has been-chosen. Since $\mathcal{Q}$ is a basis for the topology of $X$ and $\mathbb{Q}_{n-1}$ is finite and its members are closed, for each $p \notin \cup \mathbb{Q}_{n-1}$ there is a $V_{p} \in \mathbb{Q}$ such that $p \in V_{p}$ and $V_{p} \cap\left(\cup \mathbb{Q}_{n-1}\right)=\varnothing$. Since $E^{n}(x)=\alpha_{0} \times \alpha_{1} \times \cdots \times \alpha_{n-1}$ $\times\{x(n)\} \times\{x(n+1)\} \times \cdots$, and each $\alpha_{i}$ is compact, $E^{n}(x)$ is compact. Hence $E^{n}(x)$ contains a finite subset $p_{0}, p_{1}, \ldots, p_{k}$ such that $\left\{V_{p_{i}} \mid i \leq k\right\}$ covers $E^{n}(x)$ - $\left(\cup Q_{n-1}\right)$. For $i \leq k$ and $j<n$ let $g_{i j}=\left\{K \mid K\right.$ is $V_{p_{i}}(j)$ or a maximal subinterval of $\left.\alpha_{j}-V_{p_{i}}(j)\right\}$. If $j<n$ define $g_{j}=\left\{\bigcap_{i \leq k} K_{i} \mid K_{i} \in g_{i j}\right\}$. And if $j \geq n$ define $J_{j}=\bigcap_{i \leq k} V_{p_{i}}(j)$. Then define $\mathscr{B}_{n}=\left\{J \mid J \subset V_{p_{i}}\right.$ for some $i \leq k$ and $J=\prod_{j \in \omega_{0}} J_{j}$ where $J_{j} \in g_{j}$ for $\left.j<n\right\}$.

Let $I_{n}=\bigcap_{V \in \mathbb{Q}_{n-1} \cup \mathscr{B}_{n}} V(n)$. Since $V \in \mathbb{Q}_{n-1} \cup \mathfrak{B}_{n}$ implies $V \cap E^{n}(x) \neq \varnothing$, $x(n) \in I_{n}$. For $V \in \mathbb{Q}_{n-1} \cup \mathscr{B}_{n}$, define ${ }^{n} V \in \mathscr{B}$ by ${ }^{n} V(i)=V(i)$ for $i \neq n$ and ${ }^{n} V(n)=I_{n}$. Then define $\mathbb{Q}_{n}=\left\{{ }^{n} V \mid V \in \mathbb{Q}_{n-1} \cup \mathscr{B}_{n}\right\}$; clearly $\mathbb{Q}_{n}$ has the desired properties. 
For all $r$ and $n$ in $\omega_{0}$ and $V \in \mathbb{Q}_{n}$, define $V^{n r}=V(0) \times \cdots \times V(n-1) \times I_{n}$ $\times I_{n+1} \times \cdots \times I_{n+r} \times V(n+r+1) \times \cdots ;$ and define $V^{n}=V(0) \times \cdots$ $\times V(n-1) \times I_{n} \times I_{n+1} \times \cdots$. Let $\mathcal{H}=\left\{V^{n} \mid n \in \omega_{0}\right.$ and $\left.V \in \mathbb{Q}_{n}\right\}$. Clearly $V$ $\supset V^{n 0} \supset V^{n 1} \supset \cdots \supset V^{n}$, so $V \in \mathbb{Q}_{n} \subset \mathbb{Q}$ implies $V^{n} \in \mathbb{Q}$. By an easy induction on $r, V^{n r} \in \mathbb{Q}_{n+r}$. Thus the fact that, for all $m \in \omega_{0}$, the members of each $\mathbb{Q}_{m}$ are disjoint, yields that $\mathcal{T}$ is a collection of disjoint members of $Q_{\text {. }}$

Define $Z=\left\{p \in X \mid\right.$ for some $n \in \omega_{0}, p(m) \in I_{m}$ for all $\left.m>n\right\}$. Clearly $x \in Z \in \mathcal{E}$. We prove $Z=\cup \mathcal{H}$ and this proves the lemma.

Clearly each term of $\mathcal{H}$ is contained in $Z$, so we only need prove $Z \subset \cup \mathcal{F}$. Suppose $p \in Z$. There is an $n \in \omega_{0}$ with $p(m) \in I_{m}$ for all $m \geq n$. Let $q$ be the point of $E^{n}(x)$ with $q(m)=p(m)$ for $m<n$ and $q(m)=x(m)$ for $m \geq n$. Then $q \in V \in \mathbb{Q}_{n}$. But also $p \in V$ and $p \in V^{n} \in \mathcal{H C}_{\text {. So }} Z \subset \cup \mathcal{H}$.

Lemma 3. If $\mathbb{Q} \in \Omega$, then $[\mathrm{CH}]$ there is a set of disjoint members of $\mathscr{E}(\mathbb{Q})$ covering $X$.

Proof. Define a one-to-one function $f: \omega_{1} \times \omega_{1} \rightarrow \omega_{1}$ such that $f(\beta, \alpha)>\beta$ for all $\beta$ and $\alpha ; f$ need not be onto.

For each countable ordinal $\beta$ we define sets $\mathcal{K}_{\beta}$ and $\mathscr{K}_{\beta}$ by transfinite induction. Our induction hypotheses are:

(1) $\mathcal{H}_{\beta} \subset \mathcal{E}(\mathscr{Q})$ and $\mathscr{K}_{\beta} \subset \mathcal{E}$.

(2) $\mathcal{H}_{\beta} \cup \mathscr{K}_{\beta}$ is a disjoint cover of $X$ and no term of $\mathcal{H}_{\beta}$ intersects a term of $\mathscr{K}_{\beta}$.

(3) $\rho<\beta$ and $V \in \mathscr{T}_{\rho}$ implies $V \in \mathscr{H}_{\beta}$.

(4) $\rho<\beta$ and $V \in \mathscr{K}_{\beta}$ implies there is a $U \in \Re_{\rho}$ with $U \supset V$.

We use some functions in the definitions and we define these before beginning the induction. Suppose $\beta<\omega_{1}$ and suppose $\left\{\mathcal{K}_{\rho} \mid \rho \leq \beta\right\}$ and $\left\{\mathcal{K}_{\rho} \mid \rho \leq \beta\right\}$ have been defined satisfying the induction hypotheses. Then define a function $g_{\beta}:\left(K_{\beta}-\{\varnothing\}\right) \times \omega_{1} \rightarrow \mathcal{L}$ as follows. Suppose $\varnothing \neq W \in \mathcal{K}_{\beta}$. If $\rho \leq \beta$, by (4), there is a $W(\rho) \in \mathcal{K}_{\rho}$ such that $W(\rho) \supset W$. By (2), the terms of $\mathcal{K}_{\rho}$ are disjoint so $W(\rho)$ is uniquely determined. For $\rho \leq \beta$ and $n \in \omega_{0}$, define $g_{\rho n}=\{I \mid I$ is either $W(\rho)^{*}(n)$ or a maximal interval of $\left.\alpha_{n}-W(\rho)^{*}(n)\right\}$. Let $g_{n}=\left\{\bigcap_{\rho \leq \beta} I \mid I\right.$ $\left.\in g_{\rho n}\right\}$ and $g=\left\{\prod_{n \in \omega_{0}} I_{n} \mid I_{n} \in g_{n}\right\}$. Since $\{\rho \leq \beta\}$ is countable, the cardinality of $g$ is at most that of the continuum. Hence $[\mathrm{CH}]$ there is a function $g_{W}$ from $\omega_{1}$ onto $g_{\text {. For } \alpha} \in \omega_{1}$ define $g_{\beta}(W, \alpha)=g_{W}(\alpha)$.

We are now ready to begin our induction. Define $\mathcal{K}_{0}=\varnothing$ and $\mathscr{K}_{0}=\{X\}$.

Assume $\mathcal{T}_{\beta}$ and $\mathcal{K}_{\beta}$ satisfying the induction hypotheses have been defined for all $\beta<\gamma$ where $0<\gamma<\omega_{1}$.

We first define $\mathscr{K}_{\gamma}$ and $\mathscr{K}_{\gamma}$ in the case $\gamma=\delta+1$ for some $\delta<\omega_{1}$. Observe that part of our assumption in this case is that $g_{\beta}$ has been defined for all $\beta \leq \delta$. Let $Q_{\delta}=\left\{U \in \mathscr{K}_{\delta} \mid\right.$ there are $\alpha \in \omega_{1}, \beta<\delta$, and $W \in \mathcal{K}_{\beta}$ such that $f(\beta, \alpha)=\delta$, $U \subset W$, and the top of $g_{\beta}(W, \alpha)$ belongs to $\left.U\right\}$.

Fix $U \in Q_{8}$. Since $f$ is one-to-one, $\alpha$ and $\beta$ are uniquely determined. By (2) there can be at most one $W \in \Re_{\beta}$ such that $U \subset W$. So $g_{\beta}(W, \alpha)$ and the top $t_{U}$ of $g_{\beta}(W, \alpha)$ are uniquely determined by $U$. 
Choose $Z \in \mathcal{E}(\mathbb{Q})$ with $t_{U} \in Z$ as guaranteed by Lemma 2; keep in mind that $Z$ is a function of $U$. Define $H_{U}=H \cap Z$. For $n \in \omega_{0}$ define $\mathscr{F}_{n}=\{I \cap J \mid I$ is either $U^{*}(n)$ or a maximal subinterval of $\alpha_{n}-U^{*}(n)$ and $J$ is either $Z^{*}(n)$ or a maximal subinterval of $\left.\alpha_{n}-Z^{*}(n)\right\}$. Then define $\mathscr{F}=\left\{\prod_{n \in \omega_{0}} F_{n} \mid F_{n} \in \mathscr{F}_{n}\right.$ and, for infinitely many $\left.n, F_{n} \nsubseteq Z^{*}(n)\right\}$. Let $\mathcal{K}_{U}=\left\{\cup_{p \in F} E(p) \mid F \in \mathscr{F}\right.$ and $\left.F \subset U\right\}$. The terms $\mathscr{K}_{U}$ are disjoint and their union is $U-Z$.

Now define $\mathcal{K}_{\gamma}=\mathcal{K}_{\delta} \cup\left\{H_{U} \mid U \in Q_{\delta}\right\}$ and $\mathcal{K}_{\gamma}=\left(\mathcal{K}_{\delta}-Q_{\delta}\right) \cup \cup\left\{\mathcal{K}_{U} \mid U\right.$ $\left.\in Q_{\delta}\right\}$. Using only the preceding paragraph and the facts that $\delta+1=\gamma$, the induction hypotheses are satisfied for $\beta<\gamma, Q_{\delta} \subset \mathcal{K}_{\delta}$, and that for each $U \in Q_{\delta}$, a unique term $t_{U}$ of $U$ has been chosen, it is easy to check that the induction hypotheses hold for $\gamma$. The messy definitions of $g_{\beta}$ and $Q_{\delta}$ are only used later. But we need $\mathcal{K}_{\gamma}$ and $\mathcal{K}_{\gamma}$ chosen in this complicated way in order to prove the lemma.

If $\gamma$ is a limit ordinal, define $\mathcal{K}_{\gamma}=\cup_{\beta<\gamma} \mathcal{F}_{\beta}$ and $\mathscr{E}_{\gamma}=\left\{\cap_{\beta<\gamma} V_{\beta} \mid V_{\beta} \in \mathcal{K}_{\beta}\right\}$. By Lemma $1, \varnothing \neq V \in \mathcal{L}_{\gamma}$ implies $V$ is the union of a set $\mathcal{K}_{V}$ of disjoint members of $\mathscr{E}$. Define $\mathscr{K}_{\gamma}=\cup\left\{\mathscr{K}_{V} \mid V \in \mathcal{L}_{\gamma}\right\}$. The induction hypotheses clearly thus hold for $\gamma$.

Define $\mathscr{K}=\cup_{\beta<\omega_{1}} \mathcal{H}_{\beta}$. The members of $\mathcal{X}$ are certainly disjoint terms of $\mathscr{E}(\mathbb{Q})$ so the lemma is proved if $\mathcal{H}$ covers $X$.

Assume $p \in X-\cup \mathcal{K}_{C}$. Then for each $\beta<\omega_{1}$, there is a unique $U_{\beta} \in \mathcal{K}_{\beta}$ with $p \in U_{\beta}$. For $n \in \omega_{0}$ and $\beta<\omega_{1}$ let $g_{\beta n}=\left\{I \subset \alpha_{m} \mid I\right.$ is either $U_{\beta}^{*}(n)$ or a maximal subinterval of $\left.\alpha_{n}-U_{\beta}^{*}(n)\right\}$. For $n \in \omega_{0}$ and $\beta<\omega_{1}$, let $I_{\beta n}$ be the term of $g_{\beta n}$ to which $p(n)$ belongs, and for each $\delta<\omega_{1}$, let $J_{\theta n}=\bigcap_{\beta \leq \delta} I_{\beta n}$. Define $J_{\delta}=\Pi_{n \in \omega_{0}} J_{\delta n}$. Clearly $p \in J_{\delta} \subset U_{\delta}$, and $\beta<\delta$ implies $J_{\delta} \subset J_{\beta}$. Let $t_{\delta}$ be the top of $J_{\delta}$; that is, $t_{\delta}=\Pi_{n \in \omega_{0}} \sup \left\{x(n) \mid x \in J_{\delta}\right\}$. Clearly $t_{\delta} \in J_{\delta}$, and $\beta<\delta$ implies $t_{\delta}(n) \leq t_{\beta}(n)$ for all $n$. Since $\omega_{1}$ and $\alpha_{n}$ are well ordered, there is, for each $n \in \omega_{0}$, a smallest $\beta_{n}<\omega_{1}$ such that $t_{\beta_{n}}(n)=\inf \left\{t_{\beta}(n) \mid \beta<\omega_{1}\right\}$. Define $\beta=\sup \left\{\beta_{n} \mid n\right.$ $\left.\in \omega_{0}\right\}$. Then $t_{\beta}=t_{\delta}$ for all $\delta>\beta$. Look again at the definition of $g_{\beta}$. If $U_{\beta}=W$, then $\rho \leq \beta$ yields $U_{\rho}=W(\rho)$ and $g_{\rho n}=g_{\rho n}$. So $J_{\beta n} \in g_{n}$ and $J_{\beta} \in g_{0}$. Hence, $J_{\beta}=g_{\beta}\left(U_{\beta}, \alpha\right)$ for some $\alpha<\omega_{1}$. Let $f(\beta, \alpha)=\delta$; look at the definition of $\mathcal{T C}_{\gamma}$ and $\mathscr{K}_{\gamma}$ in the case $\gamma=\delta+1$. Clearly $U_{\delta} \in Q_{\delta}$ and $t_{U_{b}}=t_{\delta}$. Thus $t_{\delta}$ belongs to a term of $\mathcal{H}_{\gamma}$; but this contradicts $t_{\delta}=t_{\gamma} \in U_{\gamma} \in \mathcal{K}_{\gamma}$. Hence $\mathcal{H}_{\text {covers } X}$.

Theorem 1. The continuum hypothesis implies the box product of countably many o-compact ordinals is paracompact. In fact every open cover of such a product has a refinement consisting of disjoint open-closed sets.

Proof. By Lemma 0 we assume a space $X=\prod_{n \in \omega_{0}} \alpha_{n}$ where each $\alpha_{n}$ is compact. Let $\mathcal{G}$ be an open cover of $X$. Define $Q=\{V \in \mathscr{B} \mid$ for some $G \in \mathcal{G}, V$ $\subset G$. Obviously $\mathbb{Q} \in \Omega$. So, by Lemma $3,[\mathrm{CH}]$ there is a set $\mathcal{H}$ of disjoint members of $\mathscr{E}(\mathfrak{Q})$ covering $X$. For $H \in \mathcal{K}$, let $\mathbb{Q}_{H}$ denote a set of disjoint members of $\mathcal{Q}$ whose union is $H$. Then $\cup_{H \in X} \mathscr{Q}_{H}$ is a set of disjoint open sets refining $\mathcal{G}$ and covering $X$. 
Theorem 2. Suppose that for each $n \in \omega_{0}, \gamma_{n}$ is an ordinal and $Y$ is the box product of $\left\{\gamma_{n} \mid n \in \omega_{0}\right\}$. Suppose $\delta$ is an uncountable ordinal and $S=\left\{n \in \omega_{0} \mid\right.$ $\left.\operatorname{cf}\left(\gamma_{n}\right)=\delta\right\} \neq \varnothing$. Suppose also that $n \in \omega_{0}-S$ implies $\gamma_{n}$ is $\sigma$-compact. Define $\kappa=\sup \left\{\right.$ cardinality of $\Pi_{n \in \omega_{0}-\{s\}} \beta_{n} \mid s \in S, \beta_{n} \leq \gamma_{n}$, and $\beta_{n}$ is compact $\}$. Then $[\mathrm{CH}]$ and $\delta>\kappa$ imply $Y$ is normal.

An analogous proof shows $Y$ is collectionwise normal.

Proof. Using Lemma 0, we assume $n \in \omega_{0}-S$ implies $\gamma_{n}$ is compact. Suppose $A$ and $B$ are disjoint closed subsets of $Y$. Define $X$ to be the box product of $\left\{\alpha_{n}\right\}_{n \in \omega_{0}}$ where $\alpha_{n}=\gamma_{n}$ when $n \in \omega_{0}-S$ and $\alpha_{n}=\gamma_{n}+1$ when $n \in S$. Observe that $\alpha_{n}$ is compact for each $n$, and $Y$ is a subspace of $X$. We now use the notation set up at the beginning of $\S \mathrm{E}$ for $X$; recall $\mathscr{B}$ is a basis for $X$. Define $\mathbb{Q}=\{W \in \mathscr{B} \mid$ either $W \cap A=\varnothing$ or $W \cap B=\varnothing\}$. Lemma 4 below proves $\mathbb{Q} \in \Omega$. Then Lemma 3 proves $[\mathrm{CH}]$ there is a set $\mathcal{H}$ of disjoint members of $\mathscr{E}(\mathbb{Q})$ covering $X$. For $H \in \mathcal{H}$ let $\mathbb{Q}_{H}$ denote a set of disjoint members of $\mathbb{Q}$ whose union is $H$. Let $U=\cup\left\{W \in \mathbb{Q}_{H} \mid H \in \mathcal{H}\right.$ and $\left.W \cap A \neq \varnothing\right\}$ and $V=\cup\left\{W \in \mathbb{Q}_{H} \mid\right.$ $H \in \mathcal{H}$ and $W \cap B \neq \varnothing\}$. Then $U \supset A, V \supset B$, and $U \cap V=\varnothing$. Thus $Y$ is proved normal.

Lemma 4. Assume $y, S, \delta, \kappa, A, B, X$, and $\mathcal{Q}$ as above. Suppose $x \in X$ and $\kappa<\delta$. Then $x \in \cup Q$.

Proof. Let $R=\left\{n \in S \mid x(n)=\gamma_{n}\right\}, Z=\prod_{n \in R} \gamma_{n}, W=\prod_{n \in \omega_{0}-R}(x(n)+1)$; then $(W \times Z) \subset Y$.

If $R=\varnothing$ then $x \in Y$ and, since $A$ and $B$ are closed and disjoint in $Y$, the lemma is true. Assume $R \neq \varnothing$ for the rest of the proof. Observe that $R \neq \varnothing$ and $\kappa<\delta$ imply the cardinality of $W$ is less than $\delta$. We have two similar major cases.

Case (1). $R$ has more than one member. In this case, by the definition of $k$ and $\kappa<\delta, \gamma_{n}=\delta$ for all $n \in R$. For $\sigma<\delta$, define $z_{0}$ to be the point of $Z$ all of whose coordinates are $\sigma$ and define $Z_{\sigma}$ to be the set of all points of $Z$ all of whose coordinates are greater than $\sigma$. We use $\left\{z_{0} \mid \sigma<\delta\right\}$ and $\left\{Z_{0} \mid \sigma<\delta\right\}$ to help us choose a special ordinal $\lambda<\delta$.

Case (1a). $R \neq \omega_{0}$. In this case we wish to choose $\lambda<\delta$ such that, for all $p \in W$, one of the following hold:

(i) $\left(p, Z_{\lambda}\right) \cap B=\varnothing$ and $\left(p, z_{\lambda}\right) \in A$,

(ii) $\left(p, Z_{\lambda}\right) \cap A=\varnothing$ and $\left(p, z_{\lambda}\right) \in B$, or

(iii) $\left(p, Z_{\lambda}\right) \cap(A \cup B)=\varnothing$.

Let $P=\left\{p \in W \mid\right.$ there is a $\sigma<\delta$ such that $q \in Z_{\sigma}$ implies $(p, q) \notin A$ $\cup B$ \}. Since the cardinality of $W$ is less than $\delta$, there is a $\beta<\delta$ such that $p \in P$ and $q \in Z_{\beta}$ implies $(p, q) \notin A \cup B$. Any $\lambda$ chosen with $\beta \leq \lambda<\delta$ yields (iii) for all $p \in P$. If $W \subset P$, define $\lambda=\beta$ and (iii) holds for $p \in W$. Otherwise we have $\left(1 \mathrm{a}^{*}\right)$ or $\left(1 \mathrm{a}^{* *}\right)$.

Case $\left(\mathrm{la}^{*}\right) . W-P \neq \varnothing$ and $\delta \neq \omega_{1}$. 
Suppose $p \in W-P$ and define $\Delta_{p}=\left\{\sigma \in \delta \mid\left(p, z_{0}\right) \in(A \cup B)\right\}$.

Suppose $\left\{\sigma_{\eta} \mid \eta \in \omega_{1}\right\}$ is a monotone subset of $\delta$, and $q_{\eta} \in Z_{\sigma_{\eta}}-Z_{\sigma_{\eta+1}}$ and $\left(p, q_{\eta}\right) \in A \cup B$ for all $\eta \in \omega_{1}$. Let $\sigma=\sup \left\{\sigma_{\eta} \mid \eta \in \omega_{1}\right\}$. Since $R$ is countable, $\left(p, q_{\sigma}\right)$ is a limit point of $\left\{\left(p, q_{\sigma_{\eta}} \mid \eta \in \omega_{1}\right\}\right.$. So $\left(p, q_{\sigma}\right) \in A \cup B$ and $\sigma \in \Delta_{p}$ and only countably many $\left(p, q_{\sigma_{n}}\right)$ can belong to the one of $A$ and $B$ to which $\left(p, q_{0}\right)$ does not belong.

Thus by the preceding paragraph there is a $\sigma_{p}<\delta$ such that either $\{(p, q) \mid$ $\left.q \in Z_{\sigma_{p}}\right\} \cap A=\varnothing$ or $\left\{(p, q) \mid q \in Z_{o_{p}}\right\} \cap B=\varnothing$. Let $\delta^{*}=\{\sigma \in \delta \mid \operatorname{cf}(\sigma)$ $\left.\geq \omega_{1}\right\}$. By the preceding paragraph and the definition of $P$, since $p \in W-P$, $\Delta_{p} \cap \delta^{*}$ is a cardinality $\delta$ closed subset of $\delta^{*}$. Thus $\Delta_{p}^{\prime}=\left\{\rho \in \Delta_{p} \cap \delta^{*} \mid \rho>\sigma_{p}\right.$ and $\rho>\beta$ \} is a cardinality $\delta$ closed subset of $\delta^{*}$. Recall that $\delta$ is an uncountable ordinal and $\operatorname{cf}(\delta)=\delta$. Thus it is standard set theory that the intersection of any family of cardinality less than $\delta$ of closed subsets of $\delta^{*}$, each of cardinality $\delta$, is nonempty. Therefore, since the cardinality of $W$ is less than $\delta$, there is a $\lambda \in \bigcap_{p \in W-P} \Delta_{p}^{\prime}$. Clearly $p \in W-P$ implies (i) or (ii) holds for $\lambda$; and, since $\lambda>\beta, p \in P$ implies (iii) holds.

Case $\left(1 \mathrm{a}^{* *}\right) . W-P \neq \varnothing$ and $\delta=\omega_{1}$. Observe that cardinality less than $\delta$ means countable here. Thus $R$ is finite. So we can prove the existence of a $\delta$ with the desired properties using exactly the proof given in Case (la*) if we replace $\omega_{1}$ in the proof by $\omega_{0}$ and countable by finite. In this case $\delta=\delta^{*}$.

Case (1b). $R=\omega_{0}$. In this case we wish to choose $\lambda<\delta$ such that, for all $p \in W$, either $Z_{\lambda} \cap A=\varnothing$ or $Z_{\lambda} \cap B=\varnothing$. Such a $\lambda$ can be shown to exist using a simplified version of the argument given in Case (la) where all references to $P, \beta$, and $p$ are omitted.

Having chosen $\lambda$, we are now ready to prove the lemma in Case 1. Define $A^{\prime}=\left\{p \in W-P \mid\left(p, z_{\lambda}\right) \in A\right\}$ and $B^{\prime}=\left\{p \in W-P \mid\left(p, z_{\lambda}\right) \in B\right\}$. Clearly $A^{\prime}$ and $B^{\prime}$ are closed and disjoint in $W$. If $x^{\prime}$ is the point of $W$ such that $x^{\prime}(n)=x(n)$ for all $n \in \omega_{0}-R$, then $x^{\prime} \notin A^{\prime} \cap B^{\prime}$. Hence, for each $n \in \omega_{0}$ $-R$, there is an open and closed subinterval $I_{n}$ of $x(n)+1$ containing $x^{\prime}(n)$ such that either $A^{\prime} \cap \prod_{n \in \omega_{0}-R} I_{n}=\varnothing$ or $B^{\prime} \cap \prod_{n \in \omega_{0}-R} I_{n}=\varnothing$. For $n \in R$ define $I_{n}=Z_{\lambda}$. Then $x \in \prod_{n \in \omega_{0}} I_{n} \in \mathbb{Q}$.

Case 2. $R$ has only one member. Say $R=\{r\}$.

Since $\operatorname{cf}\left(\gamma_{r}\right)=\delta$, there is a closed subset $\left\{z_{\sigma} \mid \sigma<\delta\right\}$ of $\gamma_{r}$ such that $\sigma<\eta<\delta$ implies $z_{\sigma}<z_{\eta}$ and $\beta<\gamma_{r}$ implies $\beta<z_{o}$ for some $\sigma<\delta$. Since $R=\{r\}$, $z_{\sigma} \in Z$. Define $Z_{\sigma}=\left\{\beta \in \gamma_{r} \mid \beta>z_{\sigma}\right\}$. Then using precisely the same argument given in Case 1 after the definitions of $z_{\sigma}$ and $Z_{\sigma}$, one shows $x \in \cup Q$.

\section{BIBLIOGRAPHY}

1. M. E. Rudin, $A$ normal space $X$ such that $X \times I$ is not normal, Fund. Math. 73 (1971) 179-186. 2. The box product of countably compact many metric spaces, General Topology and Appl. 2 (1972), 293-298.

Department of Mathematics, University of Wisconsin, Madison, Wisconsin 53706 